\title{
ENERGY-ECONOMY ANALYSIS
}

Linking the Macroeconomic and Systems

Engineering Approaches

C.-O. Wene

Energy Systems Technology

Chalmers University of Technology

Göteborg, Sweden

RR-96-17

October 1996

Reprinted from Energy, Volume 21, Number 9, pp. 809-824 (1996).

INTERNATIONAL INSTITUTE FOR APPLIED SYSTEMS ANALYSIS Laxenburg, Austria 
Research Reports, which record research conducted at IIASA, are independently reviewed before publication. Views or opinions expressed herein do not necessarily represent those of the Institute, its National Member Organizations, or other organizations supporting the work.

Reprinted with permission from Energy, Volume 21, Number 9, pp. 809-824 (1996). Copyright (C)1996 Elsevier Science Ltd.

All rights reserved. No part of this publication may be reproduced or transmitted in any form or by any means, electronic or mechanical, including photocopy, recording, or any information storage or retrieval system, without permission in writing from the copyright holder.

Printed by Novographic, Vienna, Austria. 


\section{Foreword}

Three decades of debate over energy questions have demonstrated the need for a multidimensional approach to explore alternative energy futures and the viability of different energy options. The answer has been to develop increasingly sophisticated modeling tools for analyzing different disciplinary perspectives. The results, however, are not easily translated from one model to another, and sometimes two different perspectives generate conflicting results. These difficulties have been reflected in ongoing discussions on energyefficiency improvements and the costs of $\mathrm{CO}_{2}$ reductions. To consider the whole situation, special tools and procedures for interpreting results and for negotiating between different perspectives are required.

This paper describes procedures that link economic models with systems engineering models. It is based on the modeling framework used for a number of assessments including the joint IIASA and the World Energy Council (WEC) study presented in Global Energy Perspectives to 2050 and Beyond. This assessment framework includes a set of linked energy, economy, and environmental models. The macroeconomic and systems engineering models are part of this framework and provide different perspectives on the energyeconomy-technology interactions. Both models have roots in work done at IIASA in the mid-1970s.

The current report uses a common, formalized language to develop stringent linking procedures. The results indicate the value of such a formal linking methodology. The procedures translate a much-debated, key parameter describing energy-efficiency improvements in the macroeconomic model into results obtained in the systems engineering model. They also shed light on fallacies that may result from a reliance on only one perspective. The methodology illustrates how linking enhances the capacity of existing, peer-reviewed models and supports the approach IIASA has taken in using models with a proven track record for its assessment of long-term energy perspectives.

Nebojša Nakićenović

Project Leader

Environmentally Compatible Energy Strategies 


\title{
ENERGY-ECONOMY ANALYSIS: LINKING THE MACROECONOMIC AND SYSTEMS ENGINEERING APPROACHES
}

\author{
C.-O. WENE \\ Energy Systems Technology, Chalmers University of Technology, Göteborg, Sweden
}

(Received 8 June 1995)

\begin{abstract}
Informal linking or softlinking of macroeconomic and systems engineering models can provide high variety tools for joint energy-economy analysis. A necessary condition for internal control of such linking is a common, formalized language describing areas of overlap between the models. The principle of a common language is discussed and demonstrated for the softlinking of a macroeconomic model (ETA-MACRO) and a systems engineering model (MESSAGE III). Copyright (C) 1996 Elsevier Science Ltd.
\end{abstract}

\section{INTRODUCTION}

Understanding energy-economy coupling is crucial for designing energy systems compatible with sustainable economic growth. Different perspectives are leading to divergent views of this coupling. Therefore, means are needed for negotiating and integrating the different approaches. The greenhouse-gas debate provides an illustration. A central question concerns the development of energy systems compatible with both economic growth and management of the risk of climate change.

Much of the debate is focused on the links between economic growth, level of energy demands, development of the energy system to supply these demands, and technology and resource bases to support the energy system. Examples of how the links may be treated are found in Refs. 1-5. The debate is often connected with alternative analytical approaches. The approaches are distinguished by their designs and uses of various models, as well as the emphasis on technological databases. The alternative philosophies may be labelled top-down macroeconomic and bottom-up engineering. The two approaches tend to disagree on the effects of energy-efficiency improvements on future levels of energy demands. ${ }^{6,7}$ The macroeconomic approach usually leads to fewer effects on compounded future energy demands than the systems engineering approach. Some authors refer to this disagreement as the Gap. ${ }^{8}$ An interesting question is whether it is possible to identify the causes of the disagreements as either different interpretations and uses of data or differences in methodology and modelling tools.

The two approaches differ considerably in their identification of the relevant system. The bottom-up or systems engineering approach builds on detailed analysis of technological options and potentials for technical changes in the energy system. The models are focused on energy flows. With the more sophisticated systems engineering models, the complex network of resources, technologies and final users may be mapped with the desired scope and detail. Alternative energy pathways can be explored from extraction to final use. Such models are ideal tools for investigating fundamental technological changes in the energy system, including consequences for emissions, investments and cash flows. Development of systems engineering models started in the 1970s. Examples of such models for the whole energy system are found in Refs. 9-12.

In the top-down or macroeconomic approach, energy enters as a production factor. The interplay with other production factors to create economic growth is captured in production functions. The technical energy system is usually treated as a black-box that is characterized by transfer functions with elasticities describing tendencies to change the fuel mix. Price changes trigger fuel switches and alter the relation between the use of energy and other production factors, but the technologies responsible for these changes are not identified. ${ }^{13,14}$ Exceptions are Manne, ${ }^{15}$ and Manne and Richels, ${ }^{3}$ who recognize generic technologies for production of electricity and synthetic fuels and to some extent describe physical flows of energy carriers. Productivity improvements are usually specified by external parameters but have been made endogenous in the Jorgensen and Wilcoxen general equilibrium model. ${ }^{2}$ 
The macroeconomic models thus capture feedbacks between the energy system and the rest of the economy. They address the effects of changing prices on economic activity, including possible reallocation of resources that affect capital formation and economic growth. The macroeconomic models have one more important function, namely, its use helps us to avoid the reductionist fallacy, e.g. the belief that the components in the compounded energy demand will remain the same in the future. Conversely, by using the systems engineering models we avoid another fallacy, which may be called the black-box fallacy, e.g. the belief that observations of previous inputs and outputs exhaust all possible responses (i.e. possible internal states) of the energy system.

Fundamental technological changes in the energy system may involve considerable feedbacks to the rest of the economy. Conversely, evaluation of economic instruments to control the risk of climate change must include consideration of the technological response of the energy system. Linking a macroeconomic model and a systems engineering model will provide a tool for the required joint energyeconomy analysis. It should also help us to avoid both the reductionist and the black-box fallacies. For linking, it is possible to use peer-reviewed models, which avoids repeating earlier work and provides needed initial quality assurance to the efforts.

The first example of linked models was reported by Hoffman and Jorgensen. ${ }^{16}$ They linked the Brookhaven Energy System Optimisation Model (BESOM) ${ }^{17}$ with a general equilibrium model. ${ }^{18}$ Later efforts by the same group involved linking to a large input-output model. ${ }^{19}$ During the $1980 \mathrm{~s}$, investigations using linked economic and system engineering models were described by Berger et al. ${ }^{20}$ and by Yasukawa et al. ${ }^{21}$ In all of these studies, links between models are informal, i.e. the information transfer between the models is directly controlled by the user. The first example of formal linking of a macroeconomic and a systems engineering model was reported by Manne and Wene. ${ }^{5}$ The resulting energy-economy model has been used for studies of national energy systems. ${ }^{22-24}$

In this paper, I report on the informal linking (softlinking) of a macroeconomic and a systems engineering model. The purpose of the linking is to provide an integrated energy-economy modelling framework for the scenario work at the International Institute for Applied Systems Analysis (IIASA). ${ }^{25}$ This work involves systems analysis of global energy perspectives in order to identify potential environmental impacts, mitigation and adaptation strategies. The time horizon is 2100 , and the world is disaggregated into 11 regions. A number of formal models are used to achieve consistency of the qualitative analysis, but energy-economy linking is at the core of the modelling efforts.

The linking is based on two models with proven track records both in global and environmental analysis. The bottom-up systems engineering model is MESSAGE III. ${ }^{11}$ The MESSAGE family of models has been used for the past two decades in analyses of national, regional and global energy systems. The latest adaptation of the ETA-MACRO ${ }^{15}$ model is selected for the top-down macroeconomic analysis. Both models have roots in work done at IIASA in the mid-1970s. A more recent modification of ETA-MACRO is known as Global 2100. ${ }^{6}$ The adaptation of Global 2100 is referred to as $11 \mathrm{R}$ to reflect its applicability to the 11 regions of the scenario work.

Model linking has provided insights into the top-down, bottom-up controversy. To substantiate these insights, quite precise softlinking procedures have to be implemented. In the first part of the paper, I therefore develop a methodological basis for internally controlled softlinking. The methodology requires exact identification of areas where the two models overlap. Such identification is achieved by describing the two models in the common, formalized language of the Reference Energy System (RES).

The advantages and disadvantages of formal and informal linking (hardlinking and softlinking) are discussed in Sec. 3. The concept of a clearing house is introduced in order to emphasize both informal information processing and potential for learning in softlinking. The relations between scenario generation and model linking are discussed in Sec. 4. Section 5 illustrates linking for one of the 11 scenario regions. The results provide a quantitative comparison of the treatment of energy-efficiency improvements in the two models.

\section{IDENTIFYING THE RELEVANT SYSTEM: THE REFERENCE ENERGY SYSTEM IN $11 \mathrm{R}$ AND MESSAGE III}

\subsection{Model descriptions in a common language}

In the Introduction, we discussed how the macroeconomic and systems engineering approaches differ in their identification of the relevant system. But the preview also indicated areas where the identified 
systems overlap. In fact, the root of the top-down, bottom-up controversy is the claim of the modellers for such overlaps. For consistency, the softlinking between the models representing the two approaches therefore has to be internally controlled, e.g. through iterations with feedback of information between the models. This means, that it must be possible to compare model results within the overlapping areas and then decide whether the models are describing the same phenomena and the same future. The procedures for feedback and comparison will be called softlinking procedures. A necessary first step to develop such procedures is to describe the system identification made by the two models in a common, formalized language. $\dagger$

For a controllable softlinking, the common language should provide common, unambiguous measuring points where the two models should yield identical results, e.g. for energy flows. This places a strong requirement on the language: if the results from the two models at the common measuring points differ by more than a preset value, it follows from the rules of the language that the models are not linked. The language is thus the basis for quality control of the softlinking. It also should guide the development of the softlinking procedures and help explain the differences due to different model approaches (the top-down/bottom-up controversy).

There is no language that can provide both the measuring points and exhaustively describe the two models. One therefore has to settle for a minimum demand. The language should provide a full description of at least one of the models and substantial parts of the other. With these requirements, it should be possible to decide either on common measuring points or that there is no overlap and therefore the prerequisite for an internally controlled softlinking does not exist. Systems engineering provides a suitable language, namely the Reference Energy System (RES).

Figures 1 and 2 show the Reference Energy System (RES) in 11R and MESSAGE III. RES is a nested flow diagram. ${ }^{27}$ Horizontal lines indicate energy flows, and vertical lines indicate distribution systems or markets for energy carriers. In most cases an energy flow goes through an energy conversion technology, where the energy is transferred to one or more new energy carriers. In Fig. 1, an energy conversion technology is indicated by a box. For practical reasons, the conversion technologies in Fig.2 are indicated only by text strings. For the selected level of aggregation, the RES diagram shows all possible paths a unit of energy can take from primary energy to consumer.

RES can be used to give a fairly complete description of both the scope and the technological detail of a systems engineering model such as MESSAGE III (cf. Wene) ${ }^{28}$ Scope here indicates the boundaries of the system and the amount of optional technologies and alternative energy paths included in the model. The technological detail refers to the level of aggregation.

Macroeconomic relations fall outside the area of competence for the RES language. In Fig. 1, the macroeconomy is a black-box, which is also a sink for electricity and non-electric fuels furnished by the energy-supply system. For our purpose, however, it is important that the way $11 \mathrm{R}$ describes the energy-supply system can be captured in a RES. As expected, the RES for MESSAGE III has more technological detail than 11R. However, there are two important qualitative differences, which emphasize different modelling philosophies rather than the engineer's quest for details. Firstly, RES identifies a chain of energy technologies that converts, transmits and distributes energy from resource to final consumer. The consumer is linked by a chain of technologies to resources. The steps in this chain are identified in Fig. 2 as resource to primary energy to secondary energy to final energy to useful energy demand. Each step is connected with costs and energy losses. MESSAGE III describes all steps, albeit crudely for specific electric demands. 11R describes only the first step for non-electric fuels, but the two first steps for electricity. Secondly, MESSAGE III identifies different useful demands which do not specifically require electricity. Such demands will be referred to as "non-elc specific". The differentiation is necessary to investigate changes in technology to convert final energy to useful energy demands, e.g. fuel switching in the heating sector. 11R models non-electric fuels as one single production factor.

\footnotetext{
†Language is here used in the sense of means to communicate as in language games. ${ }^{26}$ Language $\mathrm{A}$ is defined as "The total set of all understood language acts refering to language A". An example of a language act is when a professor gives a student a half-finished RES asking the student to enter Integrated Coal Gasification Combined Cycle power plant into the RES. If both agree that the student has done this correctly, the language act is understood. The language act also belongs to the total set of language acts that defines the language RES.
} 


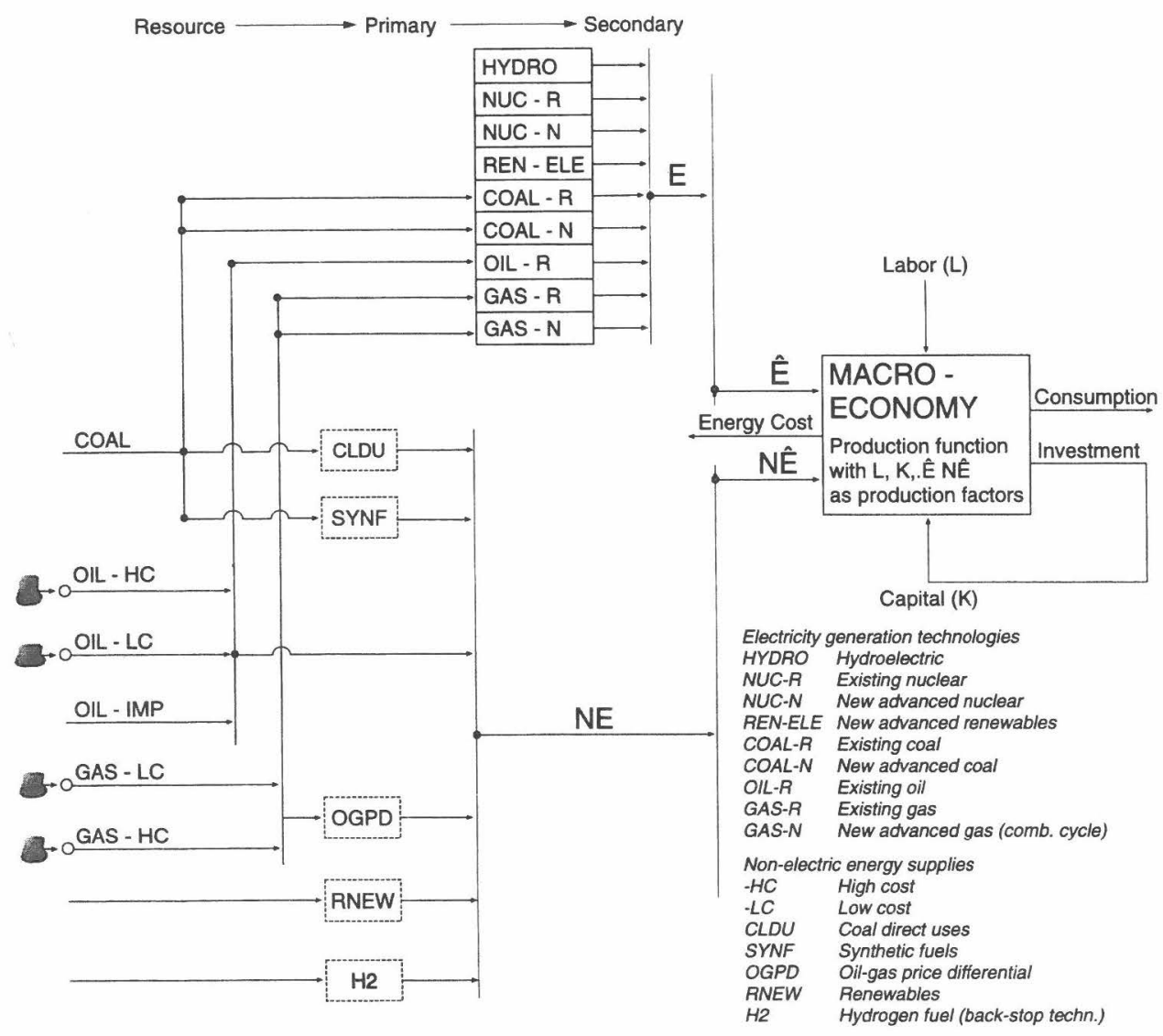

Fig. 1. Schematic diagram of $11 R$ including the Reference Energy System (RES). The RES refers to an oilimporting region. $E$ and $N E$ refer to the physical flows of electricity and non-electric fuels. $\hat{E}$ and $N \hat{E}$ are production factors. The relations between $E$ and $\hat{E}$, and between $N E$ and $N \hat{E}$ are discussed in Sec. 2.3. Dashed boxes indicate dummy technologies. These technologies are only specified by costs. No information is given about properties of interest for RES, such as conversion efficiency.

\subsection{Common measuring points}

Our RES findings are summarized in Fig. 3, which also provides the starting point for our linking. The diagram identifies the part of the RES which is common to both models. It also indicates which part of the RES is only described in MESSAGE III. The diagram shows one particular choice of common measuring points (CMP), but, in principle, these points could be chosen anywhere in the common area of the RES. The purpose of the softlinking constrains the choice. For example, for detailed sensitivity analysis the models should reproduce all the flows and capacities in the common area, and most nodes will have to be CMPs. To learn about the interactions between the two represented systems, more autonomous models and less CMPs are preferred.

Independent from the purpose of the linking, however, the set of CMPs will have some general properties that are important for a controlled softlinking. To ensure model coherence within the whole common area, the set of CMPs should react to changes anywhere within this area. We will call such a set inclusive. $\dagger$ To ensure swift convergence between the models, we would like CMPs to be independent from each other. This would simplify the procedures for model linking and quality control. 


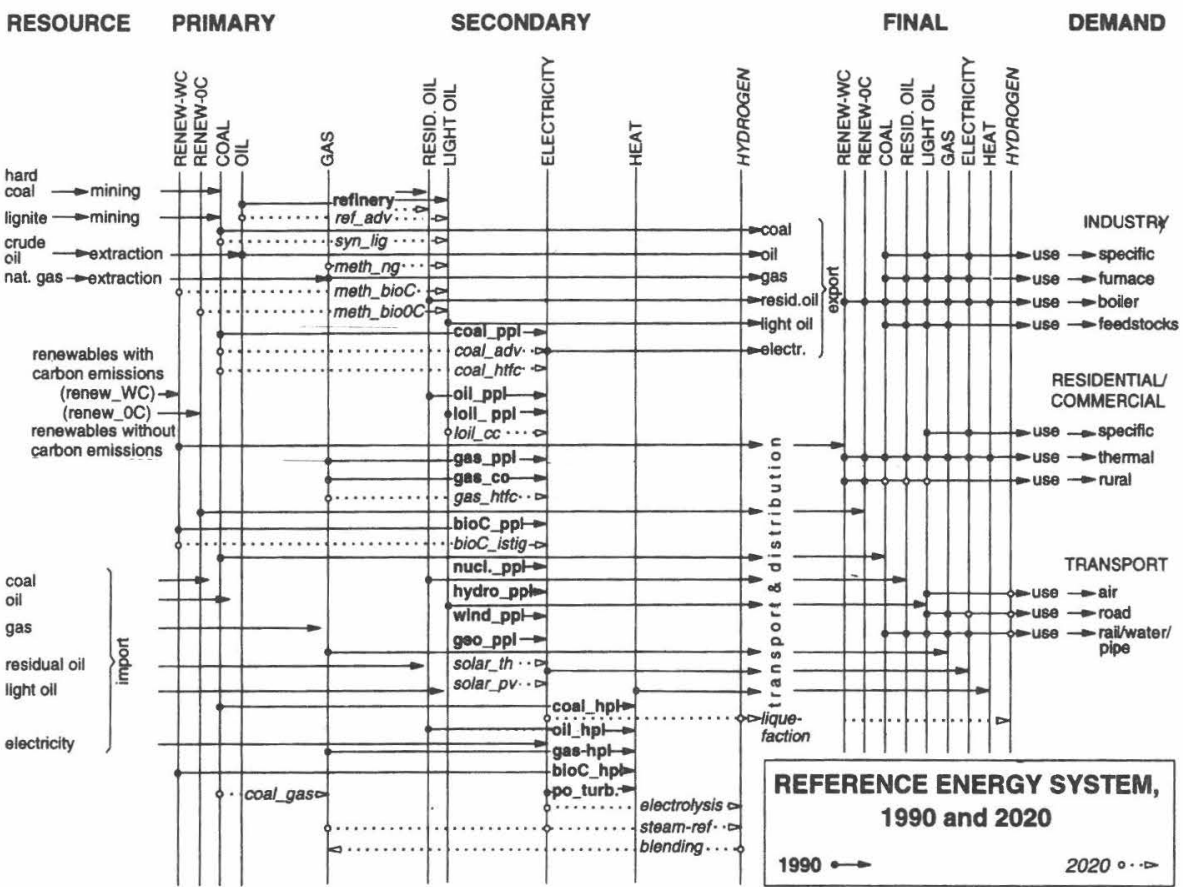

CENTRAL CONVERSION TECHNOLOGIES IN 1980

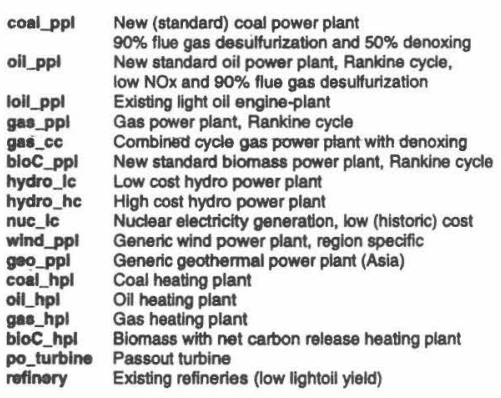

ADDITIONAL CENTRAL CONVERSION TECHNOLOGIES BY 2020

\begin{tabular}{|c|c|}
\hline oal_adv & $\begin{array}{l}\text { Advanced coal power plant, with } 99 \% \text { denoxing and } \\
\text { flue gas desulfurization; can represent supercritical, PFB, etc. }\end{array}$ \\
\hline Dal_htfc & Coal based high temperature fuel cell (internal reforming) \\
\hline & ht oll comblned cycle \\
\hline gas_httc & $\begin{array}{l}\text { High temperature fuel cell powered with natural gas } \\
\text { rejected heat avallable for co-generation }\end{array}$ \\
\hline bioc_istig & $\begin{array}{l}\text { Advanced biomass power plant: gasified biomass is } \\
\text { bumed in gas turbine plant with net carbon release }\end{array}$ \\
\hline$u c$ & Nuclear electricity generation, high cost \\
\hline solar_ & \\
\hline solar_pv & torage) \\
\hline ret_adv & ther light produ \\
\hline syn lic & Coal liquefaction and lightoil synthesis \\
\hline & \\
\hline meth_bioc & Methanol synthesis from blomass with net carbon \\
\hline meth_ & Methanol synthesis from blomass with no net carbo \\
\hline Oal 19 & \\
\hline h2_smr & Hydrogen production through steam-methane \\
\hline$h 2_{2} \theta$ & electrolysis \\
\hline hi_lit & 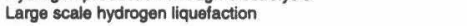 \\
\hline
\end{tabular}

Fig. 2. The RES for MESSAGE III for 1990 and 2020, adapted from Ref 25.

Such an ideal set with independent CMPs is referred to as exclusive. $\dagger$ Inclusive refers only to the common area, while exclusive apply to the complete RES.

A non-inclusive set would leave part of the common area outside softlinking. An inclusive set of CMPs is therefore a necessary condition for a controlled softlinking. Looking at the common part of the RES as a black-box, ${ }^{29}$ CMP sets consisting either of the inputs or the outputs from the common part are expected to be inclusive. It would be desirable that the set of CMPs also is exclusive. Is this possible? Unfortunately, the answer in most practical applications is no. The reason is that RES usually contains many vertical links connecting flows and technologies.

Figure 3 provides an example. The set consisting of the two points marked CMP is inclusive. How-

†o define an exclusive set, we introduce the concept of influence area. An energy flow or technology belongs to the influence area of a CMP, if a change in the flow or technology produces a change in the energy flow measured at the CMP. Two CMPs are exclusive, if there is no overlap between their influence areas. A set of CMP is exclusive if all pairs of CMPs in the set are exclusive. 


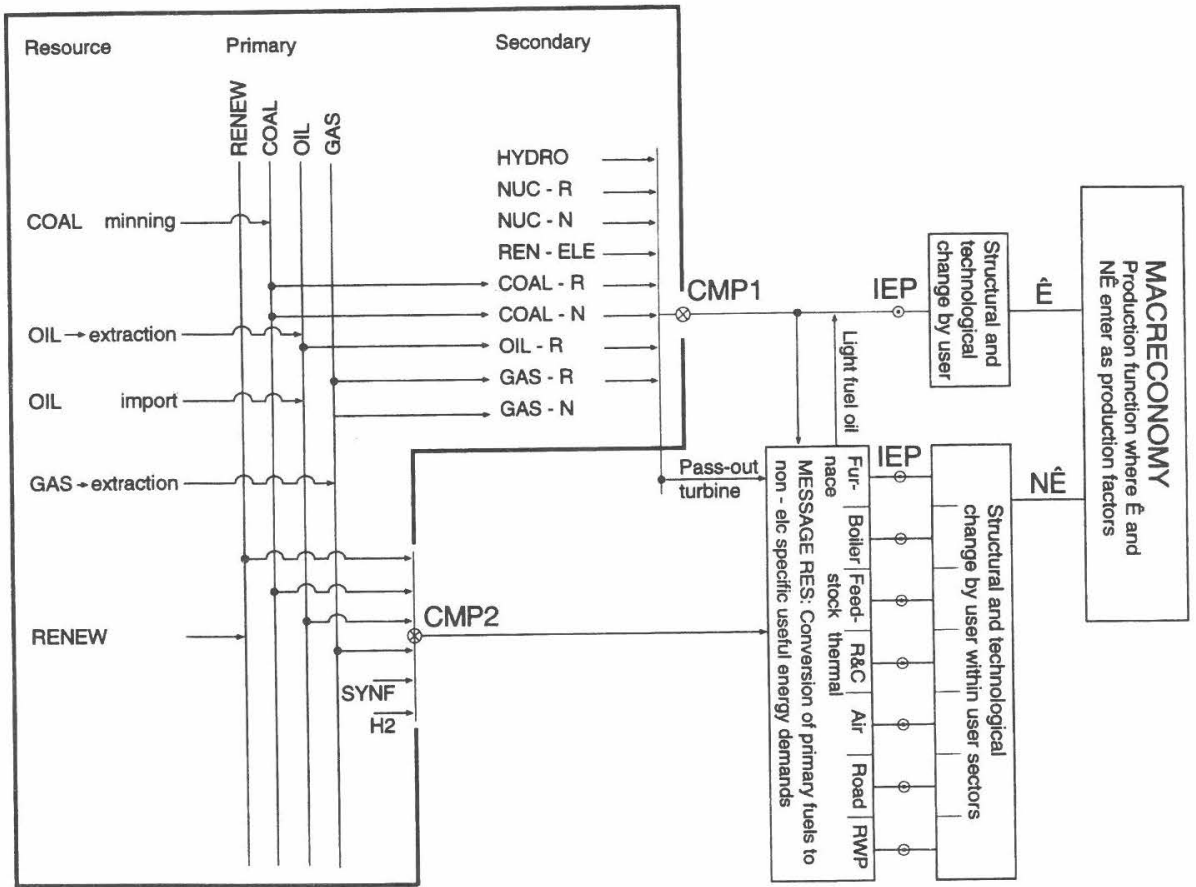

Fig. 3. The framed area shows the common RES. The common RES is obtained by mapping MESSAGE III RES on the 11R RES from Resource to common measuring points CMP. The coupling to the macroeconomy via MESSAGE III demands for commercial energy are also shown. There are cross-couplings between elc and non-elc specific demands outside the common RES. Such cross-couplings make it practically impossible to find an exclusive set of CMP and contributes to softlinking noise.

ever, switching from non-electric fuels to electricity to satisfy thermal demands will change the energy flows at both CMPs. The set of CMPs is therefore not exclusive. In fact, there are overlaps between the influence areas $\dagger$ of the two CMPs at the following conversion steps: at conversion from final to useful energy through use of electricity for thermal demands and transportation and of light fuel oil for specific demands, and at conversion from primary to secondary energy through coupled production.

The fact that the set of CMPs cannot be exclusive has important consequences. The softlinking procedures for iterating between the two models has to include corrections for overlap between influence areas. These corrections become fairly elaborate if there are large vertical flows in the RES outside the common area. The overlap between the influence areas results in softlinking noise. After several iterations, there will still be differences at the CMPs between the two models. The effect of a non-exclusive set of CMPs is further discussed in Sec. 5.

\subsection{Translating between economic and RES languages}

The previous analysis of the models is restricted by the necessity to use one common, formalized language, RES. This is sufficient to design the softlinking procedures. However, to be able to interpret the softlinking results it is necessary to understand how $11 \mathrm{R}$ describes the coupling between the physical flows of electricity and non-electric fuels and the production factors electricity and non-electric fuels. For this an economic language is necessary.

The production factors in $11 \mathrm{R}$ are capital, labour, electricity and non-electric fuels. The market share between electricity and non-electric fuels is determined by an external parameter, the value share, which in the model runs described in Sec. 5 has been kept constant over the whole studied period. The total use of electricity and non-electric fuels is determined by another external parameter, the elasticity of 
substitution between the capital-labour aggregate and the energy aggregate in the production function. For details see Manne and Richels. ${ }^{3}$

We assume that the physical energy flows $E$ and $N E$ in Fig. 3, and the energy-production factors $\hat{E}$ and $N \hat{E}$ remains identical throughout the period. With fixed relative prices between production factors, the demand for electricity and non-electric fuels will then grow at the same rate as GDP. But, according to Fig. 3, there may still be changes between the points marked CMP and the box called Macroeconomy. At this box, electricity and non-electric fuels are interpreted as production factors. Between CMP and Macroeconomy lies a major part of the MESSAGE RES and there are also possibilities for structural and technological change within user sectors. Technology R\&D and life style could be the agents of non-price-induced changes. The relations between the physical flows and the production factors are now re-interpreted ${ }^{3}$ as

$$
\begin{aligned}
E(p) & =\hat{E}(p) \times \prod_{i=1}^{i=p}[1-\operatorname{AEEI}(E, i)]^{\Delta}, \\
N E(p) & =\hat{E}(p) \times \prod_{i=1}^{i=p}[1-\operatorname{AEEI}(N E, i)]^{\Delta},
\end{aligned}
$$

where $p$ is the model period and $\Delta$ the length of the period (10 years in our case). For the base year $1990, p=0$ and $E(0)=\hat{E}(0), N E(0)=N \hat{E}(0)$. The new parameter is autonomous energy efficiency improvement (AEEI), and is introduced to capture changes between the CMPs and the production function at constant energy prices measured at the CMPs. $\dagger$

The analysis provides one important conclusion: it is possible to translate AEEI at least partially into RES-language. The MESSAGE model can be run with constant prices for electricity and for the fuels passing through the common measuring point for non-electric fuels in Fig. 3 . The changes in efficiency outside the common part of the RES is then the RES contribution to AEEI. The remaining part of AEEI is attributed to structural and technological changes within the sectors identified by MESSAGE III.

At changing prices, changes in the energy system are a result both of price, the production function and whatever driving forces behind the AEEI. Without a model for the user sectors, it does not seem possible to disentangle and translate the effects of economic parameters into RES language for this case.

\section{LINKING PROCEDURES}

\subsection{Soft-linking and hard-linking}

The discussion about a common language for system identification has been focussed on requirements for softlinking. In softlinking, the processing and transfer of the information passed between models is directly controlled by the model users. The users evaluate the results from the models and decide if and how the inputs of each model should be modified to bring the two sets of results more in line with each other, i.e. how to make the models converge. Softlinking therefore involves two different modes of information processing, namely, by using formal models and by using linking procedures. The latter mode always includes an informally evolving, judgemental part.

In order to decide on convergence, there must be a set of common measuring points CMP, as discussed in the previous section. To avoid ad hoc decisions, strict procedures for output-input processing have to be set up, using the CMP to control the linking. Before discussing the design of softlinking procedures, a comparison with formal model linking is useful to further our understanding of the advantages and disadvantages of softlinking.

In formal linking or "hardlinking", all information processing and all transfer of information between the models is formalized and usually handled by computer programs. In areas where the models overlap an algorithm may be used to negotiate results. Usually, however, one model is given strict control over the results, and the other model is set up to reproduce the same results. In a computer model, the subordinated parts are simply substituted for the corresponding parts of the controlling model.

$†$ This statement means that the price of $E$ and $N E$ will be reduced by a factor equal to the inverse of the AEEI-factors in Eqs.

(1) and (2) respectively. At constant energy prices but with non-zero AEEI, there will be take-back effects in the macroeconomy, leading to an energy demand that grows at a rate slightly greater than (GDP growth - AEEI). 
The advantages of softlinking can be summarized by practicality, transparency and learning. Likewise the advantages of hardlinking can be characterized by productivity, uniqueness and control.

Softlinking seems the most practical starting point for linking models based on different approaches. Initial investments in computer programming are kept low, and the modellers can fairly quickly obtain results for evaluation and learning. But for reasons of productivity, hardlinking is the preferred end product. As the volume of model runs increases, and more model users become involved, more resources are needed to retain the quality of softlinked than of hardlinked models.

Hardlinking produces one unique result for each set of assumptions and data. Both assumptions and data may be well documented. The quality of the results is controlled by reviewing these assumptions and data. Softlinking often produces noise in the form of differences between the results of the models for energy flows, prices and technologies.within the common region. Noise control is complicated because most of the useful sets of common measuring points turn out to be non-exclusive. Due to softlinking noise, uncertainty analysis becomes very difficult. In spite of stringent procedures, each case of softlinking contains an element of human judgement. This fact complicates outside review.

The uniqueness and ease of control in hardlinking come at a price, however. The advantage of softlinking is that at each stage of the exercise the user sees the perspective of both models, e.g. the top-down macroeconomic model and the bottom-up systems engineering model. The top-down/bottomup controversy tells us that these perspectives may lead to different results. In softlinking the differences are made explicit, as is the process of reconciling them. In this respect, softlinking can be seen as a tool for learning about the system and the implications of the two perspectives.

Softlinking and hardlinking can also be compared from the point of view of autonomy and coherence between the models. In hardlinking, model autonomy is subordinate to inter-model coherence. In softlinking, the balance between autonomy and coherence is determined through the choice of CMP and softlinking procedures. Softlinking therefore provides a high variety tool, but following the law of requisite variety, ${ }^{29}$ it consequently requires a high variety model user. This means, that a large amount of model management is needed to continuously assure the quality of the result.

\subsection{Clearing house and feedback controlled softlinking}

Figure 4 shows links between the models, scenario definition and the IIASA database. The links are shown for one region. It was emphasized in the previous section that softlinking involved different modes of information processing by using models and by using linking procedures. In Fig. 4, the concept of Clearing House is introduced to denote all activities and information processing taking place outside the models for the purpose to link the models. The Clearing House in Fig. 4 consists of model users and softlinking procedures. There is no direct connection between the two models. All information that is transferred between the models passes through the Clearing House.

Checkland $^{30}$ distinguishes between purposive and purposeful systems. A purposive system is a system designed by a purposeful system to fulfil a purpose. Only purposeful systems can reflect upon their goals and activities and change their purpose. A model can be described as a purposive, designed abstract system. But a Clearing House consists both of a designed abstract system, i.e. the softlinking procedures, and of a purposeful human activity system. It is the latter system that monitors and reflects upon the feedback from the models, designs and eventually changes the softlinking procedures.

For the softlinking of MESSAGE III and 11R, the Clearing House has three functions: design and quality control of softlinking procedures, learning about different modelling perspectives, and supporting scenario evaluations. The softlinking procedures are discussed below, and the learning in Sec. 5 .

Figure 4 indicates the feedback control of the 11R-MESSAGE softlinking. The process starts from an $11 \mathrm{R}$ run. The resulting demands for electricity and non-electric fuels are read off at the points marked CMP in Fig. 3. This information is disaggregated, corrected for effects in RES outside the common area and then fed into MESSAGE in the form of useful energy demands at the information entry points marked IEP in Fig. 3. The procedures are as follows:

The share of useful energy demands between the eight non-specific demands is part of the scenario assumptions, and the MESSAGE demands at IEP are calculated from the 11R demands using a spreadsheet model. For the first iteration with MESSAGE, the $11 \mathrm{R}$ demands are converted assuming that there is no change in efficiency in the RES between the CMPs and the IEPs. This statement means that the known base-year efficiencies for transferring energy from CMP to IEP are used throughout the whole period. For the following iterations, the previous MESSAGE run is used to correct for efficiency 


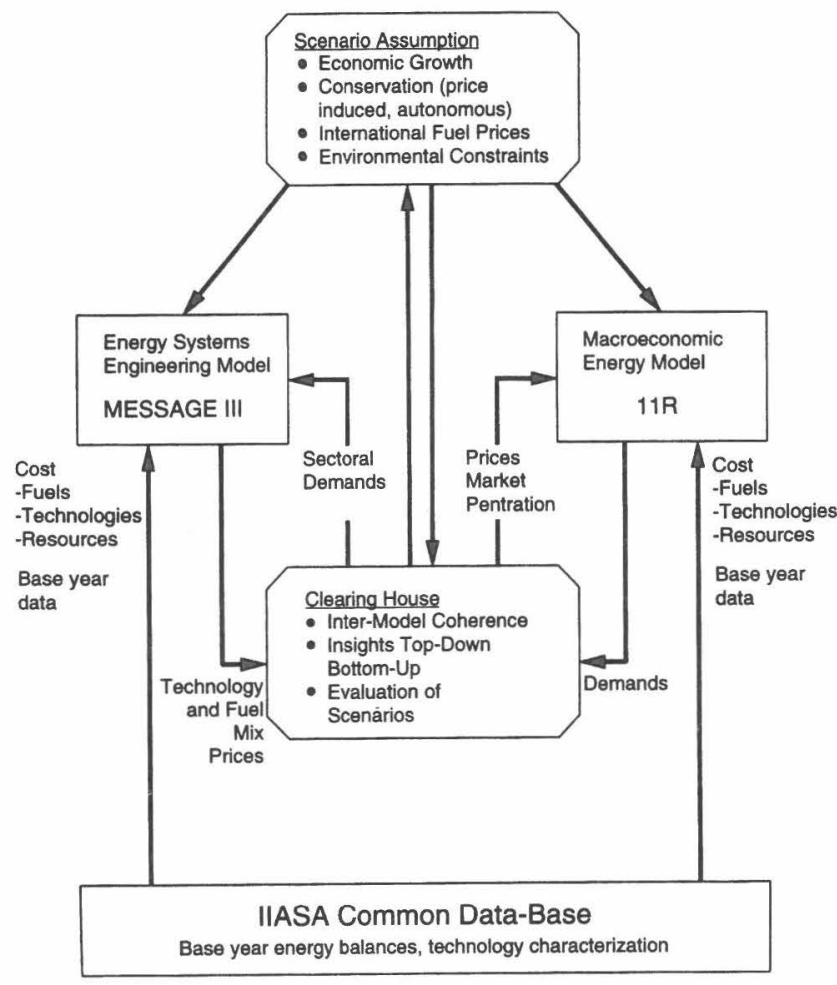

Fig. 4. The Clearing House concept.

changes in the conversion from CMPs to IEPs. Corrections also have to be made for the fact that the two CMPs are not exclusive, i.e. for the use of electricity in the non-specific sectors, for the use of light fuel oil in the elc-specific sector, and for the coupled production of electricity and heat.

The procedures used for the example in Sec. 5 allows only iterations between the Clearing House and MESSAGE III. The iterations are continued until MESSAGE III reproduces the original $11 \mathrm{R}$ values at the CMPs within a preset tolerance. It would have been quite possible to extend the set of CMPs, start iterations between the Clearing House and 11R, e.g. to make this model reproduce the fuel mix of MESSAGE. However, this would have complicated the softlinking without shedding any new lights on the top-down/bottom-up controversy.

The selected CMPs form the smallest meaningful inclusive set. $\dagger$ They therefore give a large amount of autonomy to the models. This observation is consistent with the purpose of softlinking, namely, to develop softlinking procedures for the scenario work, and to provide insights into the top-down/bottom up controversy. As experience accumulates, coherence between the models will be increased, e.g. by choosing a larger set of CMPs. The top-down/bottom-up controversy is related to energy flows after the two CMPs. The IEPs are therefore chosen as far downstream from the CMPs and as close to the Macroeconomy as possible. The meaning of the correction for efficiency changes in the MESSAGE RES after the CMPs has been elucidated previously. It is the RES contribution to AEEI.

\subsection{Other types of softlinking}

It is necessary to require an internally controlled linking for models that claim to describe the same object, e.g. the energy system. There are, however, other legitimate forms of softlinking where the models do not claim to describe the same object.

†Formally, the flows of electricity and non-electric fuels could be added and form an inclusive set with on member. It is difficult to ascribe any meaning to such a CMP from a thermodynamic point of view. 
Model chains are used to investigate causal links between different objects. One example is integrated modelling where the links between the energy-economic system and the Earth climate system is studied. ${ }^{31,32}$ Another example are the modelling chains used to investigate possible releases of radioactivity from underground repositories for nuclear waste. ${ }^{33}$ In these cases the model linking can be characterized as sequential softlinking.

Many of the concepts developed above can be taken over to sequential softlinking. Chapman et al. ${ }^{33}$ develops a common, formalized language, the Process Influence Diagram, which is used to design an Assessment Model Flowchart. In this flowchart 14 Clearing Houses are used to link 21 different models together. The Clearing Houses are of crucial importance for developing linking procedures and providing quality assurance.

\section{SCENARIO AND SCENARIO CONTROL}

Figure 4 indicates control from the scenario meta-level on the models and softlinking. The scenario level must also provide closure for any remaining discrepancies between the two models after softlinking. In the language of systems analysis, the scenario controls the boundaries of the models while the IIASA database provide system resources. Softlinking is controlled by specifying the purpose of linking. The purpose is interpreted in the Clearing House and used to find a balance between model autonomy and coherence. The balance is defined in practical terms through the choice of common measuring points (CMP) and points for entry of information from the other model (IEP).

From the point of view of the scenario level, the task for the softlinking is to check whether a suggested scenario is consistent and feasible. On this level, the rationale for probing into the topdown/bottom-up issue is less to resolve a methodological problem, but to get a preview of model behaviour and an understanding of how to interpret the answers from the softlinking process.

Closure at the scenario level has to be provided not only for softlinking within the 11 regions, but also among the regions for, e.g. traded energy carriers. To manage this task, model autonomy has to be reduced.

For the production runs, a master model has been designed using a language that is able to describe substantial parts of $11 \mathrm{R}$ and MESSAGE III. The master model interprets the scenario assumptions consistently for all the 11 regions. A Clearing House is set up on the scenario level to link the master model to the $11 \mathrm{R}$ and MESSAGE III models. Three sets of CMPs are defined: two for the linking of the master model and the 11R and MESSAGE models, respectively, and one for the linking of the 11R and MESSAGE. The CMP for the master and 11R models is the total primary energy demand, the CMPs for the master and MESSAGE III models it is the useful energy demands, and for the $11 \mathrm{R}$ and MESSAGE III the primary energy demands by source. The master model ensures coherence between regions and also reduces the softlinking noise between the $11 \mathrm{R}$ and MESSAGE III models at the individual regional levels. The price is that the regional models lose autonomy and some of their ability "to go out and find solutions for themselves". However, the reduced autonomy is consistent with the view from the scenario level of softlinked energy-economy models as tools for checking the feasibility of scenarios.

\section{SOFTLINKING AT WORK}

\subsection{An illustrative example}

The linking of MESSAGE III and 11R for China and Centrally Planned Asia has been chosen to illustrate the application of the proposed conceptual framework. Figure 5 shows the effect of softlinking at the common measuring points, i.e. total electricity production and total demands for non-electric fuels.

The pre-set criteria for softlinking prescribe that the MESSAGE III values at the CMP shall be within $3 \%$ of the $11 \mathrm{R}$ values. These criteria were met after several iterations. In Sec. 3.2., we anticipated two types of corrections to translate the $11 \mathrm{R}$ values at the CMP to useful energy demands at the MESSAGE III information entry points, IEP. It is now possible to see the magnitudes of these corrections.

The largest correction is due to changes in the compounded efficiency in the RES between the CMPs and the IEPs. Figure 6 shows these changes for the non-elc specific demand categories used in the MESSAGE model. An analysis of the model results for non-electric fuels shows that all these changes 

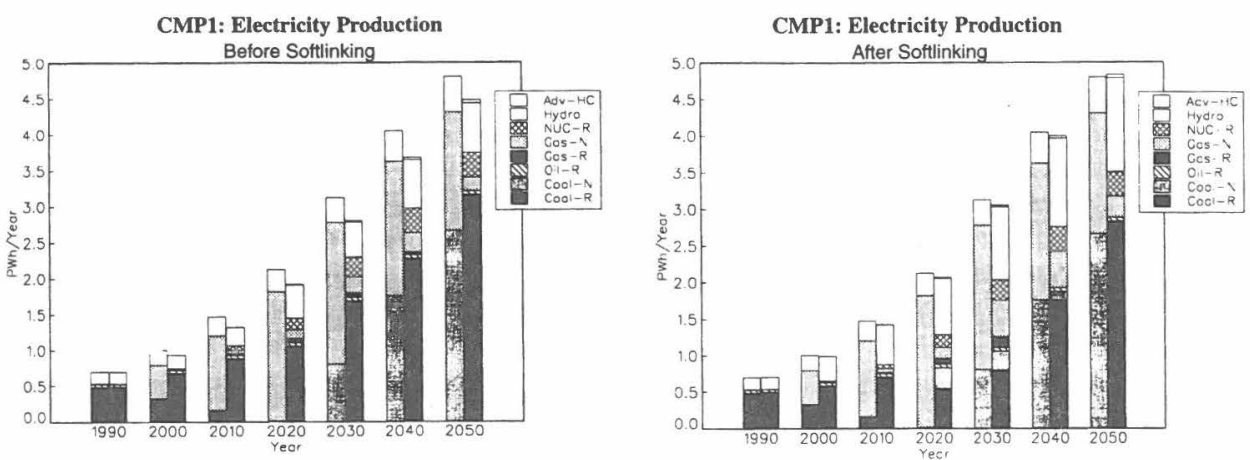

CMP2: Non-ELC Fuels Demand
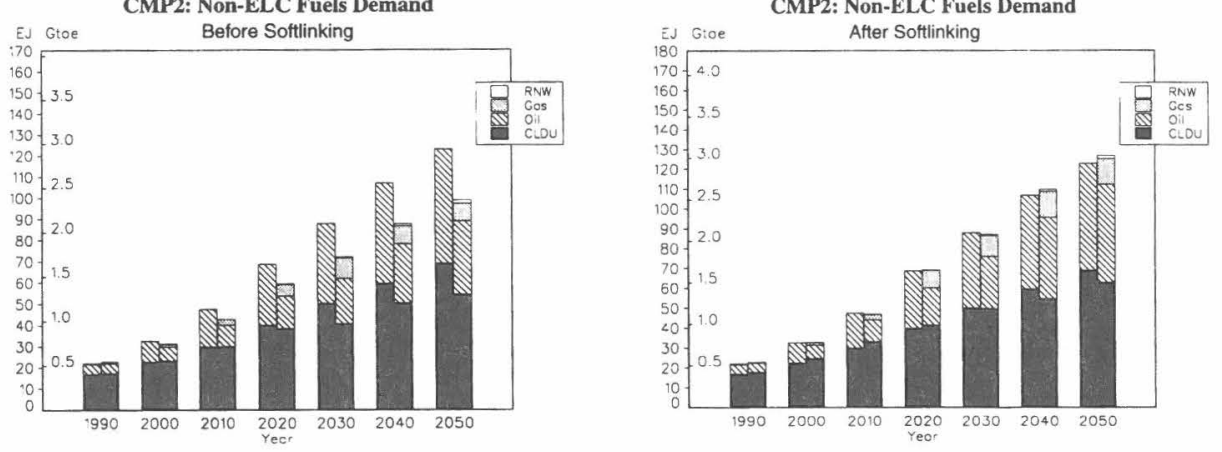

Fig. 5. $11 \mathrm{R}$ and MESSAGE III results at the two common measuring points are compared. In each diagram, left bars refer to $11 \mathrm{R}$ results and right bars to MESSAGE III results. The two left diagrams show the results before entering the Clearing House, the two right diagrams show the convergence after iteratively applying the softlinking procedures.

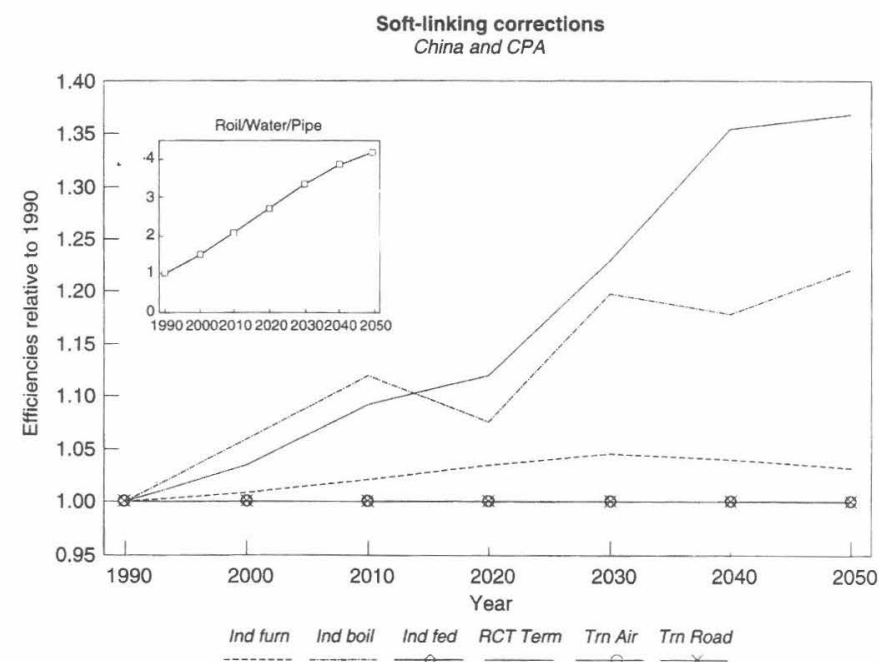

Fig. 6. Corrections for efficiency changes. The diagram shows the changes in the total efficiency in the MESSAGE III RES between the common measuring point and the information entry points for non-elc specific demands. 
are due to switching to more efficient fuel/technology combinations. The decision to switch is taken by the model, based on minimizing total cost. The changes in the electric sector are due to improvements in transmission and distribution and implicit already in the technology characterization.

The curve marked "Avg. softlink corr" in Fig. 7 shows the total efficiency correction for non-electric fuels, i.e. the average effect of fuel switching in the RES between CMP2 and IEP. Applying the efficiency correction directly to the MESSAGE III results before softlinking would already bring the MESSAGE results for non-electric fuels within the $3 \%$ interval. However, for electricity the difference between 11R and MESSAGE would still be up to $8 \%$. The fact that the set of CMPs is not exclusive has therefore a small but not negligible effect on the softlinking. In order to soft-link the two models, second order corrections due to the overlap of the influence areas of the two CMPs have to be included in the softlinking procedures. Most important is to account for the use of electricity for thermal purposes and for light oil in the elc-specific sector.

The choice of CMPs gives the models large autonomy and Fig. 5 shows that the fossil-fuels mix and the market penetration of electric technologies are different in the two models. Such type of noise can be reduced by choosing a larger set of CMPs. The efficiency correction is, however, established well above the noise level. Enlarging the set of CMPs would unnecessarily complicate procedures, if the purpose is to study the treatment of efficiency improvements in the two models.

\subsection{Interpretation of autonomous energy efficiency improvement}

The economic interpretation of the efficiency correction was discussed in Sec. 2.3. In the present case, energy prices increase substantially and change relative to each other. The average softlinking correction in Fig. 7 is then a result both of autonomous energy efficiency improvements, AEEI, and the changing share of non-electric fuels in the production. However, running the model with fixed prices for electricity and non-electric fuels will make the softlinking correction equal to the REScomponent of AEEI.

Fixed price at CMP for non-electric fuels can be obtained in several ways in MESSAGE, because this model has three fossil-fuels. Figure 7 shows the result for two quite different price schemes. The difference is small, and we conclude that with plausible fossil-fuel price schemes to keep the CMP price constant, the RES-component of AEEI implicit in the database used for this study can be fairly unambiguously defined.

Somewhat surprisingly, we find that the AEEI-curve lies above the curve showing the changes in efficiency in the original run. In the language of $11 \mathrm{R}$, this implies that increasing the price of nonelectric fuels leads to fewer efficiency improvements. Analysing the MESSAGE solution reveals the reason for the apparently contradictory result. 11R aggregates all non-electric fuels into one fuel which

\section{Non Electric Fuels: AEEI (RES-Comp)}

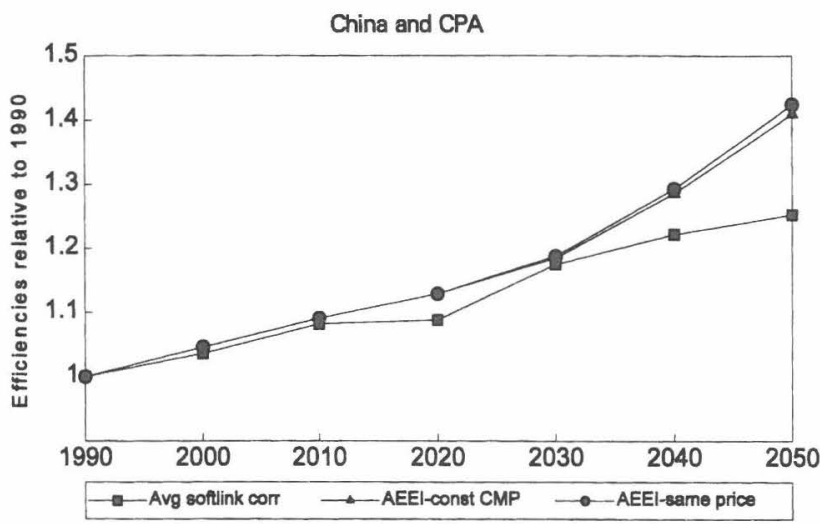

Fig. 7. Energy-weighted average changes in the total efficiency between CMP and IEPs for non-elc specific demands. The curve marked Avg softlink corr is the energy-weighted average of the corrections shown in Fig. 5. The curves marked AEEI-const CMP and AEEI-same price are results from runs with two fossil-fuels price schemes, which both give constant prices during 1990-2050 for electricity and non-electric fuels at the two CMPs. 
carries the price signal to the macroeconomy. However, most of the efficiency improvements are results of switches to more efficient fuel/technology combinations. The actual amount of switching will depend, not only on more efficient technologies becoming available, but also on the development of the relative prices between the non-electric fuels, which in our case primarily are the three fossil fuels: coal, oil and gas. The rising aggregate fuel price hides drastic changes in the relative prices. Disaggregation of the price for non-electric fuels into separate prices for the fossil-fuels therefore dissolves the contradiction. The analysis illustrates the problems of unpacking a black box.

A more detailed study of the MESSAGE results reveals that the differences between the two efficiency curves are due to different rate of investments in gas technologies. In the price schemes applied to extract AEEI from the MESSAGE database, fossil prices are artificially fixed at or close to their current values. Specifically, requiring a constant price for non-electric fuels will keep the gas price close to its low base-year value. As the more efficient gas technologies become available, they will therefore be chosen as the most cost-efficient alternatives. In the original MESSAGE runs, where price and availability of the fossil-fuels are characterized by supply curves, the present cheap gas reserves are exhausted faster than the corresponding oil and gas reserves. Gas prices therefore rise quicker than oil and coal prices, and gas technologies become less competitive. In this case, the use of gas in 2050 is less than one third of the use at constant prices.

\subsection{Bottom-up and top-down treatment of energy-economic links}

From our experiment with MESSAGE, we draw three conclusions regarding the bottom-up and topdown treatment of energy-economic links:

(a) Magnitude of AEEI. The results imply an RES-component of AEEI of $0.6 \%$ per year. The RES-component includes the effects of structural changes between the MESSAGE demand categories. The results should be compared with the assumption of $\mathrm{AEEI}=1.5$ to $1.0 \%$ /year used in the $11 \mathrm{R}$ runs. It leaves 0.9 to $0.4 \%$ /year of the AEEI to be explained by structural changes within the MESSAGE demand categories. There may also be a residual technology component because the RES does not describe technology changes within individual industrial branches. Only empirical studies will decide whether the numbers are plausible. However, softlinking makes it possible to decompose linking and pose more precise questions for these studies.

(b) Methodology vs data. Softlinking demonstrates that data can only be understood by means of a methodology. The RES-component of AEEI is to a large degree the result of increasing market penetration of already existing gas technologies, which explains why efficiency decreases as the relative price of gas increases. This explanation needs a RES-based model. On the other hand, an economic model is needed to focus on the importance of relative changes in compounded efficiency to understand the linking between primary energy availability and gross production. Softlinking does not resolve, but dissolves the dichotomy of methodology and data: data designed for one methodology may not be accessible for another methodology without the mediation of the first methodology. For example, engineering data on fuel switching and efficiency improvements must be translated or re-interpreted before entering into a macroeconomic discourse. Without such re-interpretations, most discussions between the systems engineering approach and the economic approach are meaningless. The results for the REScomponent of AEEI is an example of a re-interpretation.

(c) The reductionist vs the black-box fallacy. The RES language favours a reductionist view of demands. Demand categories are defined so that engineering data on energy conversion and distribution technologies can be entered as precisely as possible. In fact, as more demand categories are distinguished the more precise can the technology options be described. The reductionist fallacy lies in the belief, that the components of the compounded energy demands will remain the same in the foreseeable future. But within a time horizon of 50-100 years, there are historically many examples of emerging new demands. A production function oriented highly aggregated model like $11 \mathrm{R}$ allows for emerging demands. The softlinking procedures, requiring convergence between $11 \mathrm{R}$ and MESSAGE demands for electricity and non-electric fuels, provides some cure for the reductionist fallacy. But there is of course no explicit emerging demands in MESSAGE; any useful energy for emerging demands will be hidden in the 11R-corrected demands for the original categories.

The black-box fallacy refers to situations with major changes in fuel and technology prices and availability as well as in system constraints, e.g. new emission restrictions. Such changes in the system environment may activate or enhance alternative fuel-technology chains, giving the technical energy 
system properties not foreseen by a macroeconomic model. In an RES-based systems engineering model, the chains will show up as new or enhanced energy-flow paths. The situation in the oil market of 198085 provides an example. Coal was substituted for heavy oil in the electric sector, and the displaced heavy oil fraction was cracked to light products such as gasoline and light oil. Treating the technical energy system as a black-box, the system appears to produce gasoline from coal at prices slightly over $\$ 20 / \mathrm{bbl}$. A systems engineering analysis reveals, that the crude price is capped, not by emerging new technologies, but by conventional crackers and conventional coal power plant working synergistically. ${ }^{34}$

Our illustrative example in Sec. 5.1. refers to a dynamics-as-usual scenario which is a poor case for demonstrating the black-box fallacy. But the results on efficiency changes provide some insights in the mechanics of the fallacy and how to avoid it. From the RES-based model, it is evident that low relative gas prices activate the gas paths with better efficiencies, while higher relative gas prices retains more of the coal and oil. If this is considered important, the macroeconomic model can be redesigned to reflect the different efficiencies for the fossil-fuels in the non-electric fuels paths. The point to be emphasized here, however, is not that all models are infinitely malleable, but that a systems engineering model is necessary to call attention to the misrepresentation and to help decide if it has such consequences that the macroeconomic model has to be improved.

\section{CONCLUSIONS}

By identifying and starting from a common language, it has been possible to develop a rigourous methodology for feedback-controlled softlinking between a systems engineering and a macroeconomic model. Each model has a proven track record in global energy and environmental analysis. Softlinking therefore provides a quality-controlled tool with considerably enhanced capacity. Furthermore, softlinking provides at least a partial translation to a systems engineering language of an important parameter in the macroeconomic model, which provides leverage for further studies of links between energy and economy. The discussions about methodology vs data and reductionist vs black-box fallacies illustrate the bootstrapping and learning aspects of model design and use, i.e. the progress from the analystmodeller's precepts and pre-understanding of subject-matter via identification and modelling of relevant system to model solution and conclusions. Through softlinking, the analyst-modeller is urged to go back and take a second look at his/her precepts and perspective and to reassess identification and modelling of the relevant system. This learning process elucidates the conditions for efficient use of a model, i.e. the validity claims of a model. However, as one proceeds from learning to producing routine model runs, softlinking shows serious shortcomings such as softlinking noise and difficulties in maintaining the quality of the linking as the tool is transferred to new users. One remedy is to make the linking successively more formal to achieve in the end hardlinked models. Another one is to use the softlinked models as guides to develop parametrized or otherwise simplified models.

Acknowledgement-Most of this paper is based on work conducted on environmentally compatible energy strategies at IIASA. The author is grateful for many constructive suggestions to N. Nakićenović and L. Schrattenholzer, who took time to read and comment on several drafts of this paper. A. Grübler designed some of the original softlinking procedures. J. Wells helped develop the procedures further. P. Bandhari set up the original 11R case. A. Schäfer performed.all of the MESSAGE computer runs, including unavoidable troubleshooting. A. Gritsevskii computed the before-and-after softlinking figures. P. Wagner helped get manuscript and figures into a ready paper. The contents of this paper, omissions and errors, remain the sole responsibility of the author.

\section{REFERENCES}

1. W. Nordhaus, Managing the Global Commons: the Economics of the Greenhouse Effect, MIT Press, Cambridge, MA, USA (1994).

2. D. W. Jorgensen and P. J. Wilcoxen, "Energy, the Environment, and Economic Growth" in Handbook of Natural Resources and Energy Economics, Vol. III, p. 1267, A. V. Kneese and J. L. Sweeney eds, Elsevier Science Publishers, Amsterdam (1993).

3. A. S. Manne and R. G. Richels, Buying Greenhouse Insurance: Economic Cost of $\mathrm{CO}_{2}$ Emission limits, MIT Press, Cambridge, MA, USA (1992). 
4. T. Kram, "National Energy Options for Reducing $\mathrm{CO}_{2}$ Emissions, Volume 1: The International Connection, A report from the Energy Technology Systems Analysis Programme/Annex IV (1990-1993)", ECN-C-93101, Netherlands Energy Research Foundation ECN, Petten (December 1993).

5. A. S. Manne and C.-O. Wene, "MARKAL-MACRO: A linked model for energy-economy analysis", BNL47161, Department of Applied Science, Brookhaven National Laboratories, Long Island, NY, USA (1992). Reprinted in Advances in Systems Analysis: Modelling Energy-Related Emissions on a National and Global Level, p. 153, J.-Fr. Hake, M. Kleeman, W. Kuckshinrichs, D. Martinsen, and M. Walbeck eds, Konferenzen des Forschungszentrums Jülich, Band 15/1994, Jülich: Forschungszentrum, Zentralbibliotek (1994).

6. A. S. Manne and R. G. Richels, The Energy Journal 11, 69 (1990).

7. R. H. Williams, The Energy Journal 11, 35 (1990).

8. D. Wilson and J. Swisher, Energy Policy 15, 249 (1993).

9. L. G. Fishbone and A. Abilock, International Journal of Energy Research 5, 353 (1981).

10. E. van der Voort, E. Donni, C. Thonet, E. Bois D'Enhghien, C. Dechamps, and J. F. Guilmot, "Energy Supply Modelling Package EFOM-12C Mark I", CABAY, Louvain-la-Neuve, Belgium (1984).

11. S. Messner, "User's Guide for the Matrix generator of MESSAGE II, Part I: Model Description and Implementation Guide, Part II: Appendices", WP-84-71a and WP-84-71b, IIASA, Laxenburg, Austria (1994).

12. J.-Fr. Hake, P. Jagodzinski, U. Kuckshinrichs, P. Markewitz, D. Martinsen and M. Walbeck, "IKARUS: an energy-economy model to reduce energy-related greenhouse gas emissions in Germany", in Advances in Systems Analysis: Modelling Energy-Related Emissions on a National and Global Level, p. 213, J.-Fr. Hake, M. Kleeman, W. Kuckshinrichs, D. Martinsen, and M. Walbeck eds, Konferenzen des Forschungszentrums Jülich, Band 15/1994, Jülich: Foschungszentrum, Zentralbibliotek (1994).

13. J. Edmonds and J. Reilly, Global Energy: Assessing the Future, Oxford University Press, New York, U.S.A. (1985).

14. W. Nordhaus, "The 'DICE' Model: a Dynamic Integrated Climate-Economy Model of the Economics of Global Warming", Yale University, New Haven, CT, U.S.A. (1992).

15. A. S. Manne, "ETA-MACRO: a Model for Energy-Economy Interactions", in Advances in the Economics of Energy and Resources, Vol. 2, R. Pindyck ed., JAI Press, Greenwich, CT, U.S.A. (1978).

16. K. C. Hoffman and D. W. Jorgenson, The Bell Journal of Economics 8, 444 (1977).

17. E. A. Cherniavsky, "Brookhaven Energy System Optimisation Model", BNL-19569, Brookhaven National Laboratory, Upton, NY, U.S.A. (1974).

18. E. E. Hudson and D. W. Jorgenson, The Bell Journal of Economics and Management 5(2), 461 (1974).

19. P. J. Groncki and W. Marcuse, "The Brookhaven integrated energy/economy modelling system and its use in conservation policy analysis", BNL-51056, Department of Applied Science, Brookhaven National Laboratory, Upton, NY, U.S.A. (1979).

20. C. Berger, A. Haurie, G. Savard, R. Loulou, G. Lafrance, and J.-P. Surprenant, "MEDEQ-MARKAL: Un Couplage Entre Deux Modeles Techno-economique du Systeme Energetique du Quebec", R.A.I.R.O., Recherche Operationelle 21(1), (1987).

21. S. Yasukawa, O. Sato, Y. Tadokoro, T. Nagano, H. Shiraki, T. Kajiyama, Y. Nakano, and Y. Shimoyamada, "Emission Control Analysis Using the MARKAL Model of the National Energy System of Japan, Part II", in "Estimating National Costs of Controlling Emissions from the Energy System, A Report of the Energy Technology Systems Analysis Project, International Energy Agency", BNL-52252, p. 7-1, M. D. Rowe and D. Hill eds, Brookhaven National Laboratory, Upton, NY, U.S.A. (1989).

22. L. D. Hamilton, G. Goldstein, J. Lee, W. Marcuse, A. S. Manne, S. Morris, and C.-O. Wene, "MARKALMACRO: an Overview", BNL-48377, Department of Applied Science, Brookhaven National Laboratory, Upton, NY, U.S.A. (1992).

23. I. Nyström, "Improving the specification of the energy-economy link for a systems engineering model Applications for Sweden", Thesis for the Degree of Licentiate of Engineering, Report 1995:5, Energy Systems Technology, Chalmers University of Technology, Göteborg, Sweden (1995).

24. S. Kypreos, "Economic Implications of Environmental Policy for Switzerland", 4.3.04, 16th Congress of the World Energy Council, Tokyo, Japan (1995).

25. WEC (World Energy Council) and IIASA (International Institute for Applied Systems Analysis), Global Energy Perspectives to 2050 and Beyond, Report 1995, WEC, London, UK, and IIASA, Laxenburg, Austria (1995).

26. L. Wittgenstein, Philosophical Investigations, 2nd edn, Basil Blackwell, Oxford (1958).

27. W. Marcuse, L. Bodin, E. Cherniavsky, and Y. Sanborn, "A Dynamic Time Dependent Model for the Analysis of Alternative Energy Policies", in Operational Research 1975, p. 647, K. B. Haley ed., North Holland, Amsterdam, Netherlands (1976).

28. C.-O. Wene, "Exploring and Mapping: a Comparison of the IEA-MARKAL and CEC-EFOM Technical Energy System Models and the ANL Electric Utility Model", BNL-52224, Brookhaven National Laboratory, Upton, NY, U.S.A. (1989).

29. W. R. Ashby, An Introduction to Cybernetics, Chapman and Hall and University Paperbacks, London (1964).

30. P. Checkland, Systems Thinking, Systems Practice, John Wiley and Son, Chichester (1981).

31. J. Alcamo, G. J. J. Kreileman, M. S. Krol, and G. Zuidema, "Modeling the Global Society-Biosphere-Climate System, Part 1: Model Description and Testing", Water, Air, Soil Pollution, 75, (1994).

32. M. Hulme, S. C. B. Raper, and T. M. L. Wigley, "An Integrated Framework to Address Climate Change (ESCAPE) and Further Development of the Global and Regional Climate Modules (MAGICC)", in Integrative 
Assessment of Mitigation, Impacts and Adaptation to Climate Change, CP-94-9, p. 289, N. Nakićenović, W. D. Nordhaus, R. Richels, and F. L. Toth eds, IIASA, Laxenburg, Austria (1994).

33. N. A. Chapman, J. Andersson, P. Robinson, K. Skagius, C.-O. Wene, M. Wiborgh, and S. Wingefors, "Systems Analysis, Scenario Construction and Consequence Analysis Definition for SITE-94", SKI Report 95:26, Swedish Nuclear Power Inspectorate, Stockholm (1995).

34. R. J. Deam, “Coal Cost Caps Crude Prices”, Queen Mary College, University of London (September 1985). 